\title{
ON THE SUBSET SUM PROBLEM IN BRANCH GROUPS
}

\author{
ANDREY NIKOLAEV AND ALEXANDER USHAKOV
}

Stevens Institute of Technology, Hoboken, NJ, 07030, USA

e-mail address: anikolae@stevens.edu

Stevens Institute of Technology, Hoboken, NJ, 07030, USA

e-mail address: aushakov@stevens.edu

\begin{abstract}
We consider a group-theoretic analogue of the classical subset sum problem. In this brief note, we show that the subset sum problem is NP-complete in the first Grigorchuk group. More generally, we show NP-hardness of that problem in weakly regular branch groups, which implies NP-completeness if the group is, in addition, contracting.
\end{abstract}

\section{INTRODUCTION}

The study of discrete optimization problems in groups was initiated in [10], where the authors introduced group-theoretic generalizations of the classical knapsack problem and its variations, e.g., the subset sum problem and bounded submonoid membership problem. In the subsequent papers [13] and [14], the authors studied generalizations of the Post corresponce problem and classical lattice problems in groups. The investigation of knapsacktype problems in groups continued in papers $[5,8,9,6,11,12]$. The computational properties of these problems, aside from being interesting in their own right, were shown to be closely related to a wide range of well-known geometric and algorithmic properties of groups. For instance, the complexity of knapsack-type problems in certain groups depends on geometric features of a group such as growth, subgroup distortion, and negative curvature. The Post correspondence problem in $G$ is closely related to the twisted conjugacy problem in $G$, the equalizer problem in $G$, and a strong version of the word problem. Furthermore, lattice problems are related to the classical subgroup membership problem and finite state automata. We refer the reader to the aforementioned papers for details.

In this paper, we prove NP-hardness of the subset sum problem in any finitely generated weakly regular branch group. For groups with polynomial time word problem, e.g., the first Grigorchuk group, this implies NP-completeness.

Key words and phrases: Grigorchuk group, branch groups, subset sum problem, NP-completeness.

Journal of GROUPS, COMPLEXITY, CRYPTOLOGY
DOI:10.46298/jgcc.2020.12.1.6541
(C) Andrey Nikolaev and Alexander Ushakov

(c) Creative Commons 
1.1. Subset sum problem. Let $G$ be a group generated by a finite set $X=\left\{x_{1}, \ldots, x_{n}\right\} \subseteq$ $G$. Elements in $G$ can be expressed as products of the generators in $X$ and their inverses. Hence, we can state the following combinatorial problem.

The subset sum problem $\operatorname{SSP}(G, X)$ : Given words $g_{1}, \ldots, g_{k}, g$ over the alphabet $X \cup X^{-1}$, decide if

$$
g=g_{1}^{\varepsilon_{1}} \ldots g_{k}^{\varepsilon_{k}}
$$

in the group $G$ for some $\varepsilon_{1}, \ldots, \varepsilon_{k} \in\{0,1\}$.

By [10, Proposition 2.5] computational properties of SSP do not depend on the choice of a finite generating set $X$ and, hence, the problem can be abbreviated as $\mathbf{S S P}(G)$. Also, the same paper provides a variety of examples of groups with NP-complete (or polynomial time) subset sum problems. For instance, SSP is NP-complete for the following groups:

(a) the abelian group $\mathbb{Z}^{\omega}$;

(b) free metabelian non-abelian groups;

(c) wreath products of finitely generated infinite abelian groups;

(d) metabelian Baumslag-Solitar groups $B S(m, n)$ with $0 \neq m \neq n \neq 0$;

(e) the metabelian group $G B=\langle a, s, t|\left[a, a^{t}\right]=1,[s, t]=1$, as $\left.=a a^{t}\right\rangle$;

(f) Thompson's group $F$.

One can observe that in a number of the above examples, NP-completeness of SSP is a consequence of exponential subgroup distortion. Further, it is established in [16] that the latter is a sole source of NP-hardness in the case of polycyclic groups. In the present note we show that the NP-hardness of the subset sum problem for weakly regular branch groups is due to existence of abelian subgroups of arbitrarily large rank.

1.2. Zero-one equation problem. Recall that a vector $\bar{v} \in \mathbb{Z}^{n}$ is called a zero-one vector if each entry in $\bar{v}$ is either 0 or 1 . Similarly, a square matrix $A \in \operatorname{Mat}(n, \mathbb{Z})$ is called a zero-one matrix if each entry in $A$ is either 0 or 1 . Let $1^{n}$ denote the vector $(1, \ldots, 1) \in \mathbb{Z}^{n}$. The following problem is NP-complete (see [4, Section 8.3]).

Zero-one equation problem (ZOE): Given $n$ zero-one vectors $\overline{a_{1}}, \ldots, \overline{a_{n}} \in \mathbb{Z}^{n}$, decide if there exists a zero-one vector $\bar{x}=\left(x_{1}, \ldots, x_{n}\right) \in \mathbb{Z}^{n}$ satisfying $x_{1} \overline{a_{1}}+\cdots+x_{n} \overline{a_{n}}=(1,1, \ldots, 1)$, or not.

1.3. Preliminary result in branch groups. The class of branch groups was originally explicitly defined by Grigorchuk in 1997. Groups in this class possess remarkable algebraic, geometric, and analytic properties, and are studied in relation to just-infiniteness, Burnside problems, random walks, amenability, and many other topics. Geometrically, branch groups are defined in terms of action on rooted trees. We refer the reader to [2] for historic details and a thorough introduction of this class. For purposes of the present paper, we follow terminology exhibited in [2].

Let a finitely generated branch group $G$ act on a regular tree $\mathcal{T}^{(m)}, m \geq 2$. Let $\mathcal{L}_{n}$, $n=0,1,2, \ldots$, denote the $n$-th level of $\mathcal{T}^{(m)}$. Let $\psi$ be the usual embedding of the level 1 stabilizer into $G^{m}, \psi: \operatorname{St}\left(\mathcal{L}_{1}\right) \rightarrow G^{m}$. Recall that a branch group $G$ acting on the regular tree $\mathcal{T}^{(m)}$ is a regular (resp. weakly regular) branch group if $\psi$ is subdirect and there exists a finite index subgroup $K$ of $G$ such that $K^{m}$ is contained in $\psi(K)$ as a subgroup of finite (resp. perhaps infinite) index. We denote the arising embedding of $K^{m}$ into $K$ by $\chi$. 
Let $\sigma_{j}, j=0,1, \ldots, m-1$, be the embedding $\sigma_{j}: K \rightarrow K^{m}, x \mapsto(1, \ldots, 1, x, 1, \ldots, 1)$, where in the right hand side $x$ is in $(j+1)$-th coordinate. This gives us $m$ embeddings $\varphi_{j}=\chi \circ \sigma_{j}: K \rightarrow K, j=0, \ldots, m-1$.

One can notice that a (weakly) regular branch group contains $\mathbb{Z}^{\infty}$ or $\mathbb{Z}_{k}^{\infty}$ as a subgroup. In the next lemma we observe that there is such a subgroup whose first $n$ generators can be produced in polynomial time. We note that a similar construction is employed in $[1$, Section 10] (see Lemma 54 and on).

Lemma 1.1. Let a finitely generated group $G$ be a weakly regular branch group over $K$. There is

- $k$ which is an integer $k>2$ or infinity,

- a sequence $a_{1}, a_{2}, \ldots \in K$ of group elements of order $k$ such that the sum $\left\langle a_{1}\right\rangle+\left\langle a_{2}\right\rangle+\cdots \leq$ $G$ is direct, and

- a polynomial time algorithm that, given a (unary) positive integer $n$, produces $n$ elements $a_{1}, \ldots, a_{n} \in K$.

Proof. Observe that $K$ has at least one element, say $d$, of infinite order or of order $k>2$, otherwise $K$ is abelian and therefore $G$ is virtually abelian, which is imposible (see, for example, [7, Lemma 2]).

Let $p$ be the smallest integer such that $2^{p+1}-1 \geq n$. Note $p \leq \log _{2} n$. Consider the following $1+2+\ldots+2^{p} \geq n$ tuples of indices:

0 ,

100, 101,

11000, 11001, 11010, 11011,

$\ldots$,

$\underbrace{1 \ldots 1}_{j} 0 i_{1} \ldots i_{j}, \quad i_{1}, \ldots, i_{j}=0,1$

$\cdots$,

$\underbrace{1 \ldots 1}_{p} 0 i_{1} \ldots i_{p}, \quad i_{1}, \ldots, i_{p}=0,1$.

For each tuple $i_{1} \ldots i_{\ell}$ above, apply the composition $\varphi_{i_{1} \ldots i_{\ell}}=\varphi_{i_{1}} \circ \cdots \circ \varphi_{i_{\ell}}$ to the element $d \in K$. We may assume that each $\varphi_{j}$ is given in terms of (finitely many) generators of $K$, and therefore straightforward computation of each element $a_{i_{1} \ldots i_{\ell}}=\varphi_{i_{1} \ldots i_{\ell}}(d)$ takes polynomial time, since $\ell \leq 2 p+1 \leq 2 \log _{2} n+1$. Since the sum $\varphi_{0}(K)+\varphi_{1}(K) \leq K$ is direct, it follows that the $2^{p+1}-1$ elements $a_{i_{1} \ldots i_{\ell}}$ generate cyclic subgroups whose sum is direct.

\section{SSP IN $\mathbb{Z}_{k}^{\infty}$}

In this section we consider the infinitely generated group $\mathbb{Z}_{k}^{\infty}$. For algorithmic purposes, we assume that generating elements are encoded by binary strings (see, for example, [13, Section 4]).

Proposition 2.1. Let integer $k \geq 2$. The following holds.

- If $k=2$, then $\mathbf{S S P}\left(\mathbb{Z}_{k}^{\infty}\right) \in \mathbf{P}$.

- If $k>2$, then $\mathbf{S S P}\left(\mathbb{Z}_{k}^{\infty}\right)$ is $\mathbf{N P}$-complete. 
Proof. If $k=2$, then an instance $\left(\xi_{1}, \ldots, \xi_{n}, \xi\right)$ of $\operatorname{SSP}\left(\mathbb{Z}_{k}^{\infty}\right)$ is positive if and only if $\xi \in\left\langle\xi_{1}, \ldots, \xi_{n}\right\rangle$. The latter can be easily checked using linear algebra.

Let $k>2$. We claim that $\mathbf{Z O E}$ can be reduced to $\mathbf{S S P}\left(\mathbb{Z}_{k}^{\infty}\right)$. Indeed, consider an instance $\left(\overline{u_{1}}, \ldots, \overline{u_{n}}\right)$ of $\mathbf{Z O E}$, where

$$
\overline{u_{i}}=\left(u_{i 1}, \ldots, u_{i n}\right) \text { for each } i=1, \ldots n,
$$

with $u_{i j} \in\{0,1\}$. Let $b_{0} \in \mathbb{Z}_{k}^{n}$ be a sequence of zeros. For $i=1, \ldots, n$ define a sequence $b_{i} \in \mathbb{Z}_{k}^{n}$ as a sequence of zeros with 1 in $i$ th place. For each $1 \leq i \leq n$ and $v \in\{0,1\}$ define:

$$
b_{i v}= \begin{cases}b_{0} & \text { if } v=0 \\ b_{i} & \text { if } v=1\end{cases}
$$

Let $\xi_{i}$ be a concatenation $b_{i, u_{i 1}} \ldots b_{i, u_{i n}}$ and $\xi$ a concatenation $b_{n 1} \ldots b_{n 1}$. Also, define $\delta_{i} \in \mathbb{Z}_{k}^{n}$ (for $1 \leq i \leq n-1$ ) to be a sequence of zeros except for -1 in $i$ th place and 1 in $(i+1)$ th place. Finally, for each $1 \leq i \leq n$ and $1 \leq j \leq n-1$ define a sequence $\delta_{i j}$ to be concatenation of $n-1$ copies of $b_{0}$ and a single copy of $\delta_{j}$ in $i$ th place:

$$
\delta_{i j}=b_{0} \ldots b_{0} \delta_{j} b_{0} \ldots b_{0} .
$$

It is easy to see that if $\left(\overline{u_{1}}, \ldots, \overline{u_{n}}\right)$ is a positive instance of $\mathbf{Z O E}$ then $\left(\xi_{1}, \ldots, \xi_{n}, \delta_{11}, \delta_{12}, \ldots\right.$, $\left.\delta_{n, n-1}, \xi\right)$ is a positive instance of $\mathbf{S S P}\left(\mathbb{Z}_{k}^{\infty}\right)$. Conversely, suppose the latter is a positive instance of $\mathbf{S S P}\left(\mathbb{Z}_{k}^{\infty}\right)$. Inspecting the first $n$ coordinates we observe that in the solution to this instance of SSP, there must be exactly one $\xi_{i}$ with a 1 among the first $n$ coordinates; same for the second $n$ coordinates, and so on. It follows that the corresponding tuple $\left(\overline{u_{1}}, \ldots, \overline{u_{n}}\right)$ is a positive instance of ZOE.

Therefore, $\operatorname{SSP}\left(\mathbb{Z}_{k}^{\infty}\right)$ is $\mathbf{N P}$-hard when $k>2$. Since $\mathbf{S S P}(G) \in \mathbf{N P}$ for every group $G$ with polynomial time word problem we get the result.

Example 2.2. Here we give a particular example of the reduction described above. Consider an instance of $\mathbf{Z O E}$ with $n=3$ :

$$
\begin{aligned}
& (1, \quad 1,0) \text {, } \\
& (1,0,1) \text {, } \\
& (0,1,0) \text {. }
\end{aligned}
$$

Then the corresponding instance of $\mathbf{S S P}\left(\mathbb{Z}_{3}^{\infty}\right)$ is defined by a system of sequences with ... standing for an infinite sequence of zeros:

$$
\begin{array}{llll|lll|llll}
\xi_{1}= & 1 & 0 & 0 & 1 & 0 & 0 & 0 & 0 & 0 & \ldots \\
\xi_{2}= & 0 & 1 & 0 & 0 & 0 & 0 & 0 & 1 & 0 & \ldots \\
\xi_{3}= & 0 & 0 & 0 & 0 & 0 & 1 & 0 & 0 & 0 & \ldots \\
\delta_{11}= & 2 & 1 & 0 & 0 & 0 & 0 & 0 & 0 & 0 & \ldots \\
\delta_{12}= & 0 & 2 & 1 & 0 & 0 & 0 & 0 & 0 & 0 & \ldots \\
\delta_{21}= & 0 & 0 & 0 & 2 & 1 & 0 & 0 & 0 & 0 & \ldots \\
\delta_{22}= & 0 & 0 & 0 & 0 & 2 & 1 & 0 & 0 & 0 & \ldots \\
\delta_{31}= & 0 & 0 & 0 & 0 & 0 & 0 & 2 & 1 & 0 & \ldots \\
\delta_{32}= & 0 & 0 & 0 & 0 & 0 & 0 & 0 & 2 & 1 & \ldots \\
\hline \xi= & 0 & 0 & 1 & 0 & 0 & 1 & 0 & 0 & 1 & \ldots
\end{array}
$$




\section{SubSet SUM PROBLEM IN WEAKLY REgUlar BRANCH GROUPS}

Theorem 3.1. Let $G$ be a finitely generated weakly regular branch group. Then $\mathbf{S S P}(G)$ is NP-hard.

Proof. By Lemma 1.1, $G$ contains a subgroup isomorphic to $\mathbb{Z}^{\infty}$ or $\mathbb{Z}_{k}^{\infty}(k \in \mathbb{Z}, k>2)$. Recall that $\mathbf{S S P}\left(\mathbb{Z}^{\infty}\right)$ is $\mathbf{N P}$-complete by [10], and $\mathbf{S S P}\left(\mathbb{Z}_{k}^{\infty}\right), k \in \mathbb{Z}, k>2$, is $\mathbf{N P}$-complete by Proposition 2.1. By Lemma 1.1 it follows that either of those problems is $\mathbf{P}$-time reducible to $\operatorname{SSP}(G)$, therefore $\operatorname{SSP}(G)$ is $\mathbf{N P}$-hard.

The above theorem applies, for example, to the first Grigorchuk group and all so-called Grigorchuk-Gupta-Sidki groups (see [3] for a definition).

Since contracting automaton groups have polynomial time decidable word problem [15], we obtain the following corollary.

Corollary 3.2. Let $G$ be a finitely generated weakly regular contracting branch group. Then $\operatorname{SSP}(G)$ is $\mathbf{N P}$-complete.

In particular, we note that the first Grigorchuk group has an NP-complete subset sum problem.

As a final remark, we recall that the Lamplighter group also has an NP-complete subset sum problem by $[12,6]$, and the technique used in the proof of that result also involves reduction of ZOE (more precisely, the easily equivalent Exact Set Cover problem) exploiting "wide" abelian subgroups. Since both weakly regular groups and the Lamplighter group are automaton groups, this suggests the following question.

Question. Describe which automaton groups have an NP-hard subset sum problem, and which have a polynomial time subset sum problem.

\section{REFERENCES}

[1] L. Bartholdi, M. Figelius, M. Lohrey, and A. Weiß. Groups with ALOGTIME-hard word problems and PSPACE-complete compressed word problems. Preprint, 2019.

[2] L. Bartholdi, R.I. Grigorchuk, and Z. Šuniḱ. Branch groups. volume 3 of Handbook of Algebra, pages 989-1112. North-Holland, 2003.

[3] G. Baumslag. Topics in Combinatorial Group Theory. Lectures in Mathematics. ETH Zrich. Birkhäuser, 1993.

[4] S. Dasgupta, C. Papadimitriou, and U. Vazirani. Algorithms. McGraw-Hill Science, 2006.

[5] L. Frenkel, A. Nikolaev, and A. Ushakov. Knapsack problems in products of groups. J. Symbolic Comput., 74:96-108, 2016.

[6] M. Ganardi, D. König, M. Lohrey, and G. Zetzsche. Knapsack problems for wreath products. In 35th Symposium on Theoretical Aspects of Computer Science, 2018.

[7] R. I. Grigorchuk and J. S. Wilson. The Uniqueness of the Actions of Certain Branch Groups on Rooted Trees. Geometriae Dedicata, 100(1):103-116, Aug 2003.

[8] D. König, M. Lohrey, and G. Zetzsche. Knapsack and subset sum problems in nilpotent, polycyclic, and co-context-free groups. Algebra and Computer Science, 677:138-153, 2016.

[9] M. Lohrey and G. Zetzsche. Knapsack in Graph Groups, HNN-Extensions and Amalgamated Products. In 33rd Symposium on Theoretical Aspects of Computer Science, 2016.

[10] A. G. Miasnikov, A. Nikolaev, and A. Ushakov. Knapsack problems in groups. Math. Comput., 84:9871016, 2015.

[11] A. Mishchenko and A. Treier. Knapsack problem for nilpotent groups. Groups Complexity Cryptology, 9:87-98, 2017.

[12] A. Mishchenko and A. Treier. On NP-completeness of subset sum problem for Lamplighter group. Journal of Physics: Conference Series, 1050:012055, 072018. 
[13] A. G. Myasnikov, A. Nikolaev, and A. Ushakov. The Post correspondence problem in groups. J. Group Theory, 17:991-1008, 2014.

[14] A. G. Myasnikov, A. Nikolaev, and A. Ushakov. Non-commutative lattice problems. J. Group Theory, 19:455-475, 2016.

[15] V. Nekrashevych. Self-similar groups, volume 117 of Mathematical Surveys and Monographs. American Mathematical Society, Providence, RI, 16:92-95, 2005.

[16] A. Nikolaev and A. Ushakov. Subset sum problem in polycyclic groups. Journal of Symbolic Computation, 84:84-94, 2018. 